\title{
Radiation Therapy of Invasive Breast Cancer
}

\author{
Chair: \\ Richard Pötter, Alexandra Resch (Wien) \\ Participants: Wilfried Budach, Düsseldorf Peter Lukas, Innsbruck \\ Oliver J. Ott/Rolf Sauer, Erlangen Marie Overgaard, Aarhus \\ Marie-Luise Sautter-Bihl, Karlsruhe Erik van Limbergen, Leuven
}

\begin{abstract}
Question 1: Which importance do you attribute to adjuvant radiotherapy within breast-conserving treatment of invasive breast cancer? Nowadays, nearly all patients receive systemic treatment. In case of favourable prognosis, can radiotherapy be omitted for selected groups?
\end{abstract}

Budach: Adjuvant radiotherapy is an essential part of breast conserving therapy for invasive breast cancer. According to the recently published results of the meta-analysis of the EBCTCG, radiotherapy after breast conserving surgery yields a significant survival benefit. All attempts to abandon radiotherapy after breast conserving surgery resulted in a significantly increased locoregional recurrence rate. This is also true for patients above 70 years with $\mathrm{pT} 1 \mathrm{pN} 0$ hormone receptor positive tumours [Hughes et al.: N Engl J Med 2004;351: 971-7]. However, the absolute benefit in the latter group is quite small ( $\sim 5 \%$ at 7 years) and might be even smaller, if aromatase inhibitors are used instead of tamoxifen for adjuvant systemic treatment. For patients in this subgroup with limited life expectancy (e.g. $<5$ years), because of significant comorbidity, omission of radiotherapy is an option. One should, however, keep in mind that estimates on life expectancy are not very accurate and that the life expectancy of the population is continuously growing.

Lukas: Adjuvant radiotherapy after breast conserving therapy increases local control and may increase overall survival rates. Until now it cannot be omitted, even for selected groups [ABCSG 8: Lancet 2005;366:455-62, Levitt et al.: Int J Radiat Oncol Biol Phys 2003;55:645-50, Vinh-Hung et al.: Radiother Oncol 2003;67:147-58, Liljegren: Scand J Surg 2002;91:251-4].

Ott/Sauer: Adjuvant radiotherapy after breast conserving surgery had been shown to increase local control and overall survival rates in comparison to no irradiation. To date, no subgroup of patients could be identified that would not benefit from adjuvant radiotherapy, and, therefore, it should not be omitted in any case. In the subgroup of patients with favourable prognosis, systemic long-term anti-hormonal treatment is not a substitute for radiotherapy, because its effect is much more compliance dependent, and worse in terms of local control.

Overgaard: In principle I regard adjuvant radiotherapy as a standard within breast-conserving treatment of invasive breast cancer. The evidence of the benefit of radiotherapy has clearly been demonstrated in the last Oxford overview analysis published in December 2005. This study also includes several randomised trials comparing breast-conserving surgery with and without radiotherapy. Irrespective of the presence of systemic therapy radiotherapy results in a significant reduction of the local recurrence rate (3-fold) and an improvement in overall survival in the order of $5 \%$ (absolute value) after 15 years.

However, in selected groups of patients with favourable prognosis the benefit of adjuvant radiotherapy seems to be very small and might not outweigh the risks and costs of such treatment. Therefore, it is necessary to continue defining selection criteria for patients where radiotherapy can be omitted.

Sautter-Bihl: No subgroup has yet been identified where radiotherapy can be safely omitted without compromising local recurrence rates (LRR). According to the Early Breast Cancer Trialist's Collaborative Group data [EBCTCG: Lancet 2005;366:2087-106], this also holds true for older patients (absolute reduction of LRR 50-59 y: 16\%, 60-69 y: 12\%, >70 y: $11 \%$ ), small tumours (absolute reduction of LRR 1-20 mm: $15 \%$ ) and well differentiated tumours (absolute reduction of LRR: $10 \%$ ).

Van Limbergen: Adjuvant radiotherapy to the breast (whole breast or partial breast radiotherapy) is an integral part of breast conserving treatment in all patients. While the absolute

\begin{tabular}{|c|c|c|}
\hline KARGER & (C) 2006 S. Karger GmbH, Freiburg & $\begin{array}{l}\text { Univ. Prof. Dr. med. Richard Pötter } \\
\text { Univ. Prof. Dr. Alexandra Resch }\end{array}$ \\
\hline $\begin{array}{l}\text { Fax }+497614520714 \\
\text { E-mail Information@Karger.de } \\
\text { www.karger.com }\end{array}$ & $\begin{array}{l}\text { Accessible online at: } \\
\text { www.karger.com/brc }\end{array}$ & $\begin{array}{l}\text { Abteilung Strahlentherapie und -biologie, Medizinische Universität Wien } \\
\text { Währinger Gürtel 18-20, } 1090 \text { Wien, Austria } \\
\text { Tel. +43 } 1 \text { 40400-2692 Fax -2693 } \\
\text { E-mail richard.poetter@meduniwien.ac.at }\end{array}$ \\
\hline
\end{tabular}


benefits of breast radiotherapy may be small in absolute numbers in subsets of low-risk breast cancer patitents, there is no evidence that there is any subgroup in which radiotherapy could be omitted. Only in individual cases where serious competing mortality risk factors are present, radiotherapy could be omitted.

\section{Question 2: In which stage of disease do you consider adjuvant radiation therapy mandatory after radical mastectomy?}

Budach: According to the results of the 2005 EBCTCG metaanalysis postmastectomy radiotherapy results in a long-term survival benefit in all subgroups that have a $>10 \%$ risk of locoregional recurrence. Irrespective the positive effect of systemic treatments on locoregional control, radiotherapy is indicated in any of the following situations: $>3$ positive axillary lymph nodes, pT3/pT4, and R1 resection. The update of the EBCTCG meta-analysis, as shown at the ESTRO in Leipzig (October 2006), reported a significant survival advantage also for patients with 1-3 involved lymph nodes. Based on these data, I would recommend radiotherapy in this subgroup as well. Furthermore, one should discuss the potential benefits of radiotherapy with the patient, if 2 or more of the following risk factors are present: age $<40$ years, lymphangiosis or vascular invasion, G3, infiltration of the pectoral fascia, and $<5 \mathrm{~mm}$ clear resection margin.

Lukas: Adjuvant radiation therapy is mandatory (according to $\mathrm{S} 3$ recommendations) in the following cases: T3 or T4 disease with more than 4 positive lymph nodes or positive resection margins. It is recommended in case of T2 disease with lesions larger than $3 \mathrm{~cm}$ and T3, T4 tumours with resection margins $<5 \mathrm{~mm}$, carcinomatous lymphangiosis or axillary lymph node involvement.

Ott/Sauer: We consider adjuvant radiotherapy mandatory after radical mastectomy in case of tumours with a maximum diameter of $\geq 3 \mathrm{~cm}$ or involved axillary lymph nodes. In these patients adjuvant radiotherapy will lead to better local control and survival rates. For patients with preoperative chemotherapy the initial clinical stage determines further radiotherapy treatment.

Overgaard: I consider adjuvant radiation therapy mandatory after radical mastectomy in patients with $\mathrm{T} 3, \mathrm{~T} 4$, any $\mathrm{T}$ stage with positive nodes and/or invasion to deep fascia. According to the results from the two Danish radiotherapy trials and, in fact, also from the last Oxford overview of radiotherapy trials I would recommend adjuvant radiotherapy in both patients with 1-3 positive nodes and 4 or more positive nodes irrespective of the primary tumour size.

Sautter-Bihl: I consider it mandatory in case of pT3/4 tumours with more than 4 positive axillary lymph nodes and positive or close $(5 \mathrm{~mm})$ margins. Adjuvant radiation is optional in pres- ence of several of the following risk parameters: age $<40$ years, lymphatic vessel or vascular invasion, multicentricity or infiltration of the pectoral fascia.

Van Limbergen: Adjuvant radiotherapy after radical mastectomy is mandatory in cT3 or pT3 tumours, T4 (after neoadjuvant chemotherapy) or pT4, in patients with involved lymph nodes $(\mathrm{pN}+)$, in case of positive resection margins and could be considered in case of close margins or lymphatic or vascular invasion.

\section{Question 3: For which patient groups do you recommend radiotherapy of lymph nodes?}

Budach: In absence of valid data from randomised trials one has to rely on subgroup analyses. In case of more than 3 involved lymph nodes, adjuvant radiotherapy of the medial supraclavicular nodes is recommended. Excessive extracapsular growth in the axilla, and highly suspicious supraclavicular or parasternal lymph nodes do also justify radiotherapy of these nodes. Inadequate surgical staging of the axilla in highrisk patients is a further indication. A negative sentinel lymph node biopsy is considered adequate surgical staging in this regard.

Lukas: Radiotherapy of lymph nodes is recommended in the following cases: more than 3 positive lymph nodes, neck, supra- and infraclavicular with mammaria interna nodes, furthermore, if the tumour is located centrally or in the inner quadrant and if axillary nodes or sentinel nodes are involved or MRT shows enlarged lymph nodes.

Ott/Sauer: We recommend to irradiate the mammaria interna lymph node chain in case of medial or central tumour localisation, and the axillary lymph nodes if no sentinel lymph node biopsy and/or an incomplete conventional axillary lymph dissection $(<6$ nodes $)$ were performed.

Overgaard: Usually I would not recommend regional lymph node irradiation in node negative patients. However, the size and location of the primary tumour could indicate irradiation of the regional lymph nodes, for example supraclavicular and internal mammary node irradiation in medially located tumours, however, so far there are no data to confirm this approach.

Sautter-Bihl: I recommend radiotherapy of the supraclavicular fossa in case of more than 3 axillary nodes or positive nodes level III. For radiotherapy of axillary and mammaria interna nodes, see question 4.

Van Limbergen: We recommend radiotherapy to the lymph nodes after breast conserving surgery and mastectomy with axillary clearance in patients with involved lymph nodes $(\mathrm{pN}+)$. 
Question 4: In case lymph node radiotherapy is regarded as indicated, should all adjacent lymph node regions be considered for radiotherapy? When do you recommend radiotherapy of the axilla and of mammaria interna lymph nodes?

Budach: Although most patients treated in the studies included into the EBCTCG meta-analysis received radiotherapy of the axillar, the supraclavicular, and the mammaria interna nodes in addition to the chest wall irradiation, the low recurrence rates in the regional lymph nodes in studies using radiotherapy of the chest wall only, are a quite strong argument against comprehensive radiotherapy of all lymph node areas. I do recommend radiotherapy of the axilla only in case of excessive extra-capsular growth in the axilla, which is a quite rare observation. As mentioned before, inadequate surgical staging of the axilla in high-risk patients is a further indication.

We do not have valid data on the effects of mammaria interna node irradiation and have to wait for the results of the EORTC trial that randomised 4,004 patients with respect to receiving or not receiving radiotherapy of the mammaria interna and medial supraclavicular nodes. For the time being, radiotherapy of the mammaria interna nodes should be discussed, if the tumour was located in a medial quadrant and in addition axillary lymph node involvement was found. However, in case of a history of anthracyclin containing chemotherapy or ongoing or planned therapy with trastuzumab, I do not recommend radiotherapy of the mammaria interna nodes especially in left-sided tumours.

Lukas: Radiotherapy is only recommended in case of $\mathrm{R} 1$ or $\mathrm{R} 2$ margins after radical dissection (see also above). We try to avoid radiotherapy of the axilla and recommend reoperation if the axilla is not radically resected.

\section{Ott/Sauer: See answer to question 3.}

Overgaard: In a node positive patient it is relevant to consider irradiation of all adjacent lymph node areas. However, when comprehensive axillary dissection of level 1 and level 2 has been performed, only level 3 should be included in the target to reduce the risk of arm morbidity. Likewise the balance between risks and benefits by including the mammaria interna lymph nodes should be considered, especially in patients with left-sided tumours who are receiving cardiotoxic agents (anthracyclins). In general, I would recommend radiotherapy only for the top of the axilla and periclavicular nodes, if axillary dissection has been performed, and no macroscopic tumour has been left. Further, I would routinely include in radiotherapy only the mammaria interna lymph nodes in rightsided tumours and include the nodes on the left side only if they are suspicious or known to be involved.

Sautter-Bihl: Radiotherapy of the axilla is recommended only in patients with extensive extracapsular spread or infiltration of the fatty tissue. The decision is made in an interdisciplinary agreement with the surgeon. The decision for radiotherapy of the mammaria interna lymph nodes is made individually regarding the general risk profile of the patient and may be discussed in medially/centrally located tumours and $>4$ involved axillary lymph nodes.

Van Limbergen: Until the results of the EORTC IMC RT trial are known we recommend radiotherapy to the internal mammary chain in patients with positive lymph nodes and in those with centrally or medially located tumours, in case there is an uptake of tracer in the internal mammary chain at the time of the sentinel procedure, and if there is no surgical removal of this node. We recommend radiotherapy to the axilla in case of axillary involvement and if no or an insufficient axillary clearance is performed. Irradiation of the axilla is also recommended after axillary clearance in case of massive lymph node involvement (4 or more lymph nodes with capsule rupture).

\section{Question 5: Have new methods (e.g. sentinel lymph node biopsy) any impact on the indication for radio- therapy of lymph node regions, (a) negative sentinel lymph node (b) isolated tumour cells detected by immunocytochemistry in a sentinel lymph node $(p N O(s n)(i+)(<0.2 \mathrm{~mm}))(c)$ infiltration into the marginal sinus of lymph nodes, pN1 mi (micrometastasis $0.2-2 \mathrm{~mm}$ )?}

Budach: Available data do not indicate that one should change the recommendations regarding radiotherapy of regional lymph nodes. A negative sentinel node biopsy, provided the guidelines for the quality of this procedure are fulfilled, should be considered equivalent to the $\mathrm{pN} 0$ situation after removal of 10 or more axillary lymph nodes. Although the available data concerning how to proceed in case of micrometastasis in the sentinel node, regardless the location of these foci in the lymph node, is quite limited, indications of radiotherapy should not differ from the $\mathrm{pN} 0$ situation.

Lukas: Both, (a) and (b) are regarded as negative lymph node involvement; (c) is regarded as positive, an indication for radiotherapy depends on the number of positive lymph nodes.

Ott/Sauer: A negative sentinel lymph node as well as isolated tumour cells detected by immunocytochemistry in a sentinel lymph node is accepted as negative axillary staging, and does not impact target volume definition. Axillary lymph node micrometastasis ( $\mathrm{pN} 1 \mathrm{mi}$ ) is considered to be a facultative indication for the irradiation of the mammaria interna lymph node chain.

Overgaard: The introduction of sentinel lymph node biopsy could influence the indication for radiotherapy of lymph node regions in the future. It needs to be clarified whether micrometastasis or isolated tumour cells in the sentinel lymph node indicate further regional therapy, axillary dissection or 
radiotherapy. At present there are studies addressing these questions, and we will have to await the results.

Sautter-Bihl: No, these methods do not yet have an impact on the indication for radiotherapy of lymph node regions.

Van Limbergen: In case of negative sentinel node or isolated tumour cells we do not irradiate the lymph nodes unless there is tracer uptake in a non-removed lymph node, for example in the internal mammary chain. In case of $\mathrm{pN} 1 \mathrm{mi}$ we treat patients as we do in $\mathrm{pN} 1$ patients.

\section{Question 6: What should be the risk of loco-regional recurrence to recommend radiotherapy of the breast and/or of lymphatic pathways? Do you counsel against radiotherapy in certain situations with an unfavourable benefit/risk ratio, for the breast or for certain lymph node regions?}

Budach: If the risk of locoregional recurrence is below $10 \%$ at 15 years after surgery and systemic treatment, omission of radiotherapy is an option. In case of breast conserving surgery, all subgroups have a higher risk. After mastectomy, pT3/pT4 or $\mathrm{pN}+$ cases are usually associated with risk of locoregional recurrence above $10 \%$ and should at least receive chest wall radiotherapy. Indications for adjuvant radiotherapy of the lymphatic drainage of the breast are less secure and depend on the individual risk factors. In case of already existing lymph oedema of the arm, adjuvant axillary radiotherapy should not be recommended. Also it would be unwise to irradiate the supraclavicular fossa if a damage of the arm plexus is already present. Concerning the mammaria internal nodes pre-existing lung or heart disease and planned or already received potentially cardiotoxic treatments have to be taken into consideration (see also question 4). In doubt, one should better avoid adjuvant radiotherapy of the parasternal nodes.

Lukas: There is no recommendation against radiotherapy. We are only regarding the risk ratio of locoregional vs. systemic risk for choosing the schedule of treatments: high locoregional risk (tumour size and so on, see above): radiotherapy first; high systemic risk: chemotherapy first; equal risk: split course with radiotherapy in the middle of chemotherapy cycles.

Ott/Sauer: We do not counsel against radiotherapy of the breast in any case, because its local control rates are superior to all other breast conserving treatment options. Anti-hormonal treatments may satisfactorily decrease local recurrences to a certain extent at 5 years, but long-term results are lacking. Therefore, systemic therapies are no alternative for breast irradiation. Concerning the indication for lymph node region radiotherapy see question 4 .

Overgaard: I think it would be difficult to define an exact level of locoregional recurrence rate to recommend radiotherapy of breast and/or lymphatic pathways. The problem is that the absolute reduction in locoregional recurrence rate by radiotherapy is not always proportional to the improvement in overall survival. There is some indication that patients with intermediate risk of locoregional recurrences may benefit more in terms of survival compared to patients with very high risk of locoregional recurrences where the risk of disseminated disease is much more a problem. But if I should choose a number, I would recommend radiotherapy if the locoregional recurrence rate is above $10 \%$ at 10 years. On the other hand, I would counsel against radiotherapy in situations where the risk of long-term morbidity could outweigh the benefit, for example by including the internal mammary nodes in left-sided breast cancers.

Sautter-Bihl: We always recommend radiotherapy after breast conserving therapy except in case of medical contraindications. Lymph node irradiation is recommended in a risk adapted way (see also question 3 and 4).

Van Limbergen: For irraditation of the breast, the risk for recurrence and hence loss of the breast must be higher than $5 \%$. For irradiation of the thoracic wall and regional lymph nodes, a risk higher than $10 \%$ should be present. If there is a contraindication for radiation we recommend mastectomy instead of breast conserving surgery. For the lymph nodes we should omit radiotherapy if the risk for complications exceeds the benefit.

\section{Question 7: Which patients benefit most from boost treatment to the tumour bed?}

Budach: According to the updated analysis (10-year median follow-up) of the EORTC boost trial $(n>5,000)$ that was presented at the EORTC Radiation Oncology Group meeting in Groningen (Oct. 2006), the in-breast recurrence rate is significantly decreased in all subgroups. In contrast to an earlier analysis, a significant advantage was also seen in 50-60-yearold patients as well as in patients older than 60 . The hazard ratios in favour of boost irradiation were almost identical in all age groups. In absolute numbers, the benefit of a $16 \mathrm{~Gy}$ boost irradiation was higher the younger the patients. Based on these data, it is difficult to define subgroups that should not receive a boost radiotherapy. Elderly patients with small pN0 hormone receptor positive tumours and significant comorbidity are unlikely to live long enough to have an advantage from boost irradiation. More details will be known after the full publication of the update of this important trial. Independent of age close margins, lymphangiosis, vascular invasion, G3, and an excessive extraductal component have been shown to be associated with a higher in-breast recurrence rate. The benefit of boost irradiation will be even higher, if one or more of these factors are found.

Lukas: The following patients benefit most from boost treatment: patients below 50 years of age, or with resection mar- 
gins $<5 \mathrm{~mm}$, extensive intraductal component or lymph vessel involvement or poorly differentiated tumours (EORTC 22881: Radiother Oncol 2004;72:25-33, Romestaing et al.: J Clin Oncol 1997;15:963-8).

Ott/Sauer: A current analysis of the EORTC Trial 22881/10882 (boost vs. no boost) showed a significant decrease of in-breast recurrences with an additional boost throughout all age groups with the most impressive benefit among young patients ( $\leq 50$ years). Furthermore, we prescribe a tumour bed boost after incomplete resection, if there was an extensive intraductal component or lymph vessel invasion, in case of lymph node involvement or poorly differentiated tumours.

Overgaard: I think that there is good evidence from the two large boost trials, that boost treatment to the tumour bed is indicated in patients with high risk of recurrence in this area, e.g. patients below 50 years of age, residual tumour in resection margins and/or extensive in situ carcinoma.

Sautter-Bihl: All young patients ( $<50$ years) benefit from boost irradiation. In patients older than 50 years boost radiotherapy can be considered if one or several of the following risk factors are present: close margins, poorly differentiated tumours, extensive intraductal component, size $>2 \mathrm{~cm}$ and multifocality.

Van Limbergen: All patients benefit from a boost treatment but the absolute gain is higher in younger than in older patients. Long-term follow-up data from the boost no boost trial show a significant reduction of local recurrences also in the older patients.

\section{Question 8: Will the introduction of new imaging techniques into routine treatment planning (e.g. PET-CT, 3 Tesla MRT) result in any changes of classical radiation field arrangements, in particular with regard to target volume definition?}

Budach: The definition of a target volume for boost irradiation will be probably more accurate, if PET-CT scans and 3 Tesla MRT could be matched with the treatment planning CT. This would require data acquisition in exactly the same patient position as during the treatment planning CT. The spatial resolution of currently available MRT coils is inferior in this position, but might be sufficient for treatment planning purposes. Preliminary results of studies on a small number of patients have shown the technical feasibility. Whether this will translate into a clinical benefit for the patients remains to be shown. MRT but not PET may also be employed to better define the target volume for adjuvant whole mammary gland radiotherapy. The target volumes are likely to be considerably smaller than what is typically thought to be the mammary gland based on planning CT scans. In theory, this would result in decreased field sizes for radiotherapy and consequently would cause less acute and late side effects of radiotherapy.
Lukas: Not in the near future except perhaps for the indication of radiotherapy of internal mammarian lymph nodes. Molecular imaging may perhaps influence the whole treatment arrangement for breast cancer in some years.

Ott/Sauer: New imaging techniques may not change the current technique of whole breast irradiation, but may impact the indication to irradiate the mammaria interna lymph node chain.

Overgaard: New imaging techniques might not immediately result in changes of classical radiation field arrangements in postoperative radiotherapy after mastectomy or breast conserving surgery. One reason is that these techniques are not useful in the postoperative setting, and another reason are the limitations of radiotherapy resources, which is already a problem for this very large group of patients. However, these techniques might help in special cases with locally advanced disease (primary inoperable cases or loco-regional recurrences). Maybe these techniques could also be used for planning of radiotherapy in relation to neoadjuvant chemotherapy.

Sautter-Bihl: This is quite conceivable, especially for boost planning or in case of accelerated partial breast irradiation (ABPI), but this is not yet of practical relevance for clinical routine.

Van Limbergen: I do not think these new techniques will change the target definition. This will depend on results from further trials (IMC trial, AMAROS trial, Supremo trial, the NSABP and GEC-ESTRO APBI trials) for different patients subsets. PET-CT can be helpful in target delineation in locally advanced disease. The gain of MRI over CT as has been demonstrated in gynaecological malignancies and prostate cancer seems to be unlikely in breast cancer. For breast, thoracic wall and lymph node areas MRI will offer no more information than CT. For extension of the tumour the breast MRI can offer a better discrimination than CT, but implementation of MRI in breast treatment planning will need the development of MRI breast examination in supine instead of prone position or target delineation in prone position as is done by certain multicatheter APBI techniques.

\section{Question 9: Accelerated partial breast irradiation has recently been introduced for selected groups with early breast cancer. For which subgroups would you recommend $\mathrm{APBI}$ based on available evidence? Can APBI already be considered as a standard option outside prospective clinical trials?}

Budach: APBI is at the time not a recommended option for any patient outside a clinical trial. Although the results of a number of phase II trials are promising, one has to keep in mind that the long-term results of the only available randomised studies (Cookridge Hospital, Leeds, UK, 2005; 
Christie Hospital, Manchester 1996) showed significantly increased local recurrence rates in the partial breast radiotherapy arms of the studies. These studies included a substantial number of high-risk patients that would not be considered to be candidates for partial breast radiotherapy nowadays and are therefore disregarded by the proponents of APBI outside of clinical trials. Nevertheless, it is strongly recommended to treat patients within one of the ongoing clinical trials, but wait for the results of these studies before broad application.

Lukas: APBI can not be used in daily routine and may only be applied in prospective clinical trials. It is not at all a standard option!

Ott/Sauer: APBI with multi-catheter brachytherapy implants is a new promising treatment approach for a strictly selected subgroup of patients with favourable prognostic factors (tumour diameter $\leq 3 \mathrm{~cm}$, node negative, age $\geq 40$, no extensive intraductal componentor lymph vessel invasion and so on). APBI is still experimental and must not be performed outside prospective clinical trials.

Overgaard: I think it is too early to consider APBI as a standard option outside prospective clinical trials. APBI is not a well-defined treatment. There is a variety of different techniques, and definition of total dose fractionation and optimal target volume needs to be evaluated systematically. I think we will have to await results from comprehensive randomised trials comparing standard breast-conserving therapy with APBI with sufficient follow-up and proper evaluation of morbidity and cost.

Sautter-Bihl: ABPI is an experimental treatment and I would not recommend it until long-term data are available. By no means should it be performed outside prospective randomised clinical trials. Results of whole breast radiation are excellent concerning local control and cosmetic results, moreover, it improves survival. Therefore, any new method aiming to replace this treatment has to prove an equal effectiveness compared to this gold standard with a sufficient follow-up.

Van Limbergen: For the moment there is no level 1 evidence that APBI is a safe alternative to whole breast radiotherapy and boost. Trials are ongoing in the US and Europe and results should be awaited before implementation in routine. Possible candidates are patients older than 50 years with unifocal tumours smaller than $3 \mathrm{~cm}$, no extensive intraductal component, negative resection margins with at least a $5 \mathrm{~mm}$ free margin, no lymphovascular invasion and negative lymph nodes. Under discussion is APBI in patients older than 40 years if all the mentioned selection criteria are met. Data on invasive lobular adenocarcinomas suggest that if completely removed they also could be candidates for APBI. The same is true for ductal carcinoma in situ with an Van Nuys Prognostic Index $<8$.

\section{Participants}

Univ.-Prof. Dr. med. Wilfried Budach

Klinik u. Poliklinik f. Strahlentherapie u. Radiologie, Onkologie Universitätsklinikum Düsseldorf,

Moorenstr. 5, 40225 Düsseldorf, Deutschland

Tel. +49211 811-79 91, Fax -80 51

E-mail wilfried.budach@uni-duesseldorf.de

Dr. med. Marie Overgaard

Experimental Clinical Oncology, Department of Oncology Aarhus University Hospital,

Nørrebrogade 44, Bld. 5, 8000 Aarhus, Dänemark

Tel. +45 894925 43, Fax +4586139149

E-mail Marie@oncology.dk

Prof. Dr. med. Marie-Luise Sautter-Bihl

Klinik für Strahlentherapie

Städtisches Klinikum $\mathrm{GmbH}$

Moltkestr. 90, 76133 Karlsruhe, Deutschland

Tel.+49 7219 74-40 01, Fax -40 09

E-mail Marie-Luise.Sautter-Bihl@klinikum-karlsruhe.com

Prof. Dr. med. Rolf Sauer

Dr. med. Oliver J. Ott

Klinik und Poliklinik für Strahlentherapie

Universität Erlangen-Nürnberg

Universitätsstr. 27, 91054 Erlangen, Deutschland

Tel. +49 9131-85 -33 405, Fax -39 335

E-mail sekretariat@strahlen-med.uni-erlangen.de

Univ.-Prof. D. I. Dr. med. Peter Lukas

Universitätsklinik für Strahlentherapie - Radioonkologie

Medizinische Universität Innsbruck

Anichstr 35, 6020 Innsbruck, Österreich

Tel. +43 512 504-22800, Fax -22869

E-mail Peter.Lukas@uibk.ac.at

Prof. Dr. Erik Van Limbergen

Department of Radiation Oncology

University Hospital Gasthuisberg

Herestraat 49, 3000 Leuven, Belgium

Tel. +32 16 34-6907, Fax -6901

E-mail erik.vanlimbergen@uz.kuleuven.ac.be 